Historic, Archive Document

Do not assume content reflects current scientific knowledge, policies, or practices. 



\section{FALL OF 1902}

\section{WholesaleTrade List}

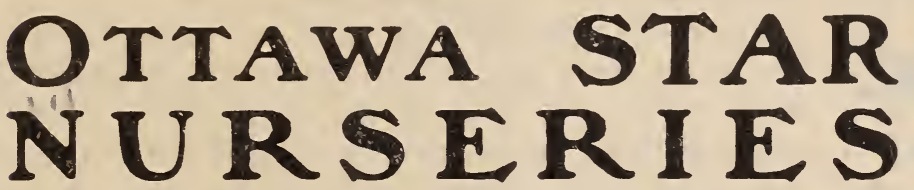

OTTAWA, \& $:$ KANSAS

F. H. STANNARD \& CO., \begin{tabular}{c} 
Successors to \\
PROPRIETORS \\
STANNRR \& \\
\hline
\end{tabular}

REFERENCES:

First National Bank, Ottawa, Kansas.

Peoples National Bank, Ottawa, Kansas.

Bank of Ottawa, Ottawa, Kansas.

Or the Mercantile Agencies.

USE - THE

" NURSERYMEN'S TELEGRAPHIC CODE" IN TELEGRAPHING.

Established 1879 


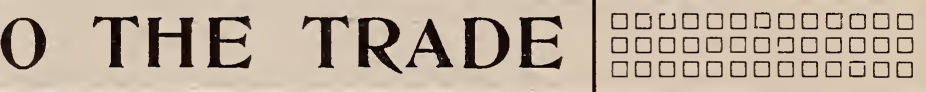

In presenting our Semi-annual Trade List for the fall of 1902 we wish to thank our many patrons for their liberal

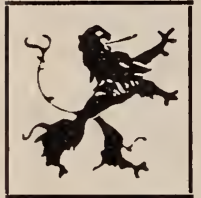

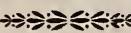
patronage during the past year, and assure them that we are much better prepared to handle their business than we have been in the past, having completed our large frostproof packing house, which is $200 \times 150$ feet and thoroughly ventilated, with a railroad switch running into the building. We respectfully solicit a share ot your patronage.

\section{LOCATION.}

Our Nurseries are situated 54 miles southwest from Kansas City, on new slope lands along the Marais des Cygnes river, the banks of which are skirted with a heavy growth of timber, affording a splendid wind break and protection to young trees. Thus we are enabled to produce clean, thrifty stock, at one and two years, suitable for the market. Our railroad and packing facilities are as good as the best, being on the main lines of the Missouri Pacific and A. T. \& S. F. railways, with a switch to our pack. ing ground.

Our Nurseries* embrace over 500 acres, closely planted, including apple, standard and dwarf pear, cherry, plum and peach, as well as a general assortment of ornamental trees and shrubs.

\section{PACKING.}

Our Packing Season begins from the 1st to the 10th of October in the fall, and from the 1 st to the 10 th of March in the spring. We pack all stock in the most thorough manner, for which a moderate charge-sufficient to cover the cost-will be made.

Boxes packed and paper lined will be charged as follows: 30 inches. square, 8 to 10 feet long, as the trees may require, at $\$ 3$ each; 24 inches square, at $\$ 2.50 ; 18$ inches square, at $\$ 1.75$. Bales at from $25 \mathrm{c}$ to $75 \mathrm{c}$ each.

\section{SHIPPING DIRECTIONS.}

Please furnish us with explicit shipping directions, mentioning the route, and whether you wish shipment by express or freight. When no particular directions are given we will use our judgment, but in no case do we assume any responsibility as to safe arrival and condition of trees and plants, after a proper shipment.

Under our present western classifications, we are entitled to a lower freight rate by releasing to a value of $\$ 5$ per 100 pounds. When not instructed to the contrary, we will release accordingly. 


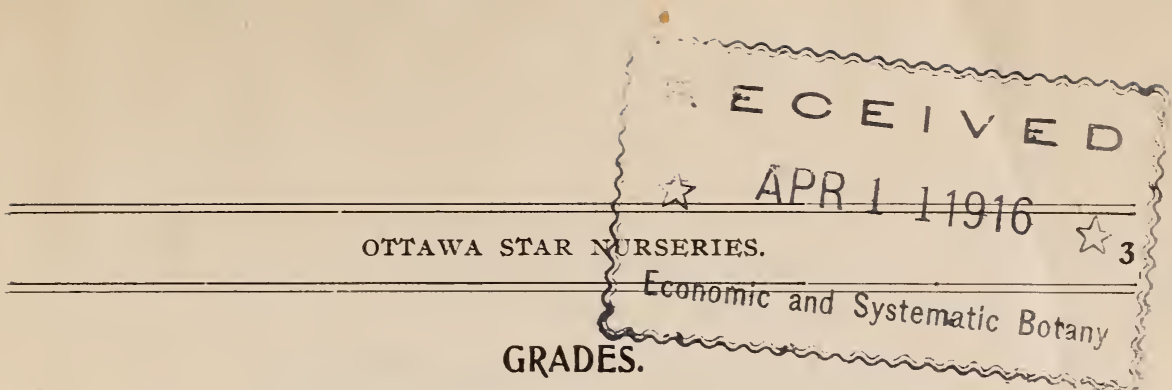

We grade carefully; in some cases by caliper, others by height; when by both, the caliper must govern. The lower number is included and the higher number excluded. For instance, in a grade of four to five feet, four-feet trees are included, but five-feet trees go into the next grade higher. If plump five-feet trees are wanted, the order must be for five to six feet. When desired, we will send samples to prospective customers.

\section{TERMS.}

Cash, or satisfactory security before shipment. Remit by Kansas City or eastern exchange, or express money order. Orders to be sent C. O. D. must be accompanied by at least one-fourth of the amount of the order.

To prompt-paying customers the usual 30 to 60 days will be allowed from the date of shipment; but where the time is not especially agreed upon, 30 days is to be understood. Interest will be charged upon all overdue accounts. Should prices be changed, either higher or lower, during the season, the prices ruling at the time the order is made are expected to stand.

When the order amounts to $\$ 50$ or over, the articles will be figured at thousand rates.

Prices are subject to change without notice.

\section{GUARANTEE OF GENUINENESS.}

While we exercise the greatest diligence and care to have all our trees and plants true to label, and hold ourselves in readiness on proper proof to replace all trees that may prove untrue to labels, free of charge or refund the amount paid, it is mutually understood and agreed to between the purchaser and ourselves, that our guarantee of genuineness shall in no case make us liable for any sum greater than originally received.

\section{A Health Certificate will be furnished with each shipment.}

\section{F. H. STANNARD \& CO., Ottawa, Kansas.}

KANSAS EXPERIMENT STATION,

E. A. Popenoe, Official Entomologist. Y

Ottawa, Kansas, Sept. 4th, 1902

Chis is to Certify, That I have this day made an examination of the Nursery Stock now growing on the grounds of F. H. STANNARD \& Co., Ottawa, Kansas, and that I find thereon no San Jose Scale or evidence of its former presence, and, moreover, I find that this stock is apparently in excellent condition as to growth, health, and freedom from noxious insects or fungous diseases.

E. A. POPENOE,

State Nursery Inspector. 


\section{FRUIT DEPARTMENT.}

\section{APPLES.}

Two and Three Years, Smooth.

Code Word. Per 100. Per 1000.

5 to $6 \mathrm{ft} ., 3 / 4 \mathrm{in}$. and up.....................

$\$ 850 \quad \$ 8000$

4 to $6 \mathrm{ft}, 5 / 8$ in. to $3 / 4$ in ........................ $\quad 650 \quad 6000$

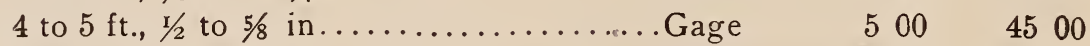

3 to $4 \mathrm{ft}$, mostly branched.............Gain $\quad 400 \quad 3500$

Varieties marked with a star will be furnished in such proportion in assortment as our stock will permit, and if supplied alone will be charged for at one cent per tree additional for the two upper grades.

Arkansas Black

Autumn Strawberry

*Alexander

*Bailey Sweet

Baldwin

Bellflower

Ben Davis

Benoni

Clayton

*Cooper's Early White

Chenango Strawberry

Duminie

*1)uchess of Oldenburg

*Early Harvest

Fall Pippin

Fameuse

Flora Belle

Gano

General Grant (Crab)

Grimes' Go!den Pippin

Golden Sweet

Hatas

Huntsman's Favorite

*Hibernal

Hyslop (Crab)

Ioiva Blush

Jonathan

Lawver
Longfield

Lowell

Loy

Limber Twig

Maiden Blush

Mann

Martha (Crab)

Mammoth Black Twig

McAfee's Nonesuch

* McIntosh Red

Mc.Mahon's White

Minkler

Missouri Pippin

Northern Spy

Northwestern Greening

Penn. Red Streak

Pewaukee

Plum Cider

* Rambo

*Red Astrachan

*Red June

Red Romanite

Red Winter Pearmain

Red Beitigheimer

Red Siberian (Crab)

Rome Beauty

Roman Stem
Rawles' Genet

* Rhode Island Greening

Salome

Scott's Winter

Smith's Cider

*Spitzenberg

Stark

Springdale

Summer Queen

* Sweet Bough

*Talman Sweet

*Tetofsky

Twenty-ounce Pippin

Transcendant (Crab)

Utter's Red

Vandevere Pippin

Wagoner

Walbridge

*Wealthy

Willow Twig

White Arctic (Crab)

White Winter Pearmain

*Whitney No. 20

Winesap

*Wolf River

York Imperial

*Yellow Transparent

BISMARCK -3 cents extra per tree. 


\section{CHERRIES ON MAHALEB.}

Two Years.

Code Word. Per 100.

Per 1000.

No. $I, 4 \frac{1}{2}$ to 6 feet, $3 / 4$ in. and up........Gangway $\$ 1800 \$ \$ 18000$

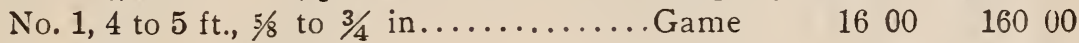

3 to $4 \mathrm{ft}$., $1 / 2$ to $5 / 8$ in..................................... 140013000

3 to $4 \mathrm{ft}$., $3 / 8$ to $1 / 2$ in........................... 120011000

Those varieties of Cherry marked with star are 5 cents additional.

* Black Tartárian

*Black Heart

Dyehouse

Early Richmond

Empress Eugene

English Morello

*Gov. Wood

*Knight's Early Black
Late Duke
Leib
Louis Philippe
Montmorency.
May Duke

*Knight's Early Black Late Duke

Louis Philippe

May Duke
*Napoleon

Olivet

Ostheimer

*Rock Port

Wragg

*Yellow Spanish

We have a good assortment of Cherry in all grades at Dansville, $\mathrm{N}$. Y., and will furnish them at that point at one cent less per tree than above prices.

\section{PEACH.}

One Year.

Code Word Per $100 \quad$ Per 1000

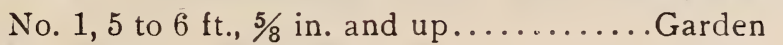

700

6500

No. 1,4 to $5 \mathrm{ft} ., 9-16$ to $5 / 8$ in............ (iargle

$600 \quad 5500$

3 to $4 \mathrm{ft}$., $7-16$ to 9.16 in............... Garland

$500 \quad 4500$

3 to $4 \mathrm{ft}$., under $716 \mathrm{in..............Garlic} 450 \quad 40 \mathrm{co}$

2 to 3 feet...................................... $350 \quad 3000$

Amsden

Alexander

Arkansas Traveler

Bokara No 3

Champion

Crawlord's Early

Crawford's Late

Coolidge's Favorite

Crosby

Elberta

Early Rivers

Early York

Emma

Foster
Fitzgerald

Foxe's Seeding

Greensboro

Gold Drop

Hale's Early

Heath Cling

Heath Free

Hill's Chili

Horton's Rivers

Kalamazoo

Lemon Free

Lemon Cling

Lewis Seedling
Mt. Rose

Old Mixon Free

Stump the World

Salway

Smock

Sneed

Susquehanna

Triumph

Wonderful

Waterloo

Wager

W'heatland

Wright's Seedling

\section{ALIMOND ON PEACH.}

One Year. 


\section{PEARS.}

Two Years - Standard.

Code Word

Jer 100

Per 1000

5 to $\% \mathrm{ft}$., No. $1,3 / 4$ in and up........... Gait $\$ 1300 \quad \$ 12000$

5 to $6 \mathrm{ft}$., No. $1,5 / 8$ to $3 / 4$ in............ Gale

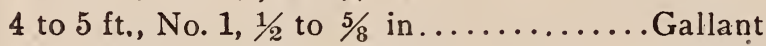

$1100 \quad 10000$.

$31 / 2$ to $5 \mathrm{ft}$., mostly branched............Gallon

$900 \quad 8000$

$700 \quad 60.00$.

Bartlett

Beurre de Anjou

Keiffer

Garber

Manning's Elizabeth
Howell

Clapp's Favorite

Lawrence

Flemish Beauty

Wilder

Lincoln

Koonce

Sheldon

Lawson

Rutter

Seckel

\section{Dwarf.}

\begin{tabular}{|c|c|c|c|}
\hline $\begin{array}{l}4 \text { to } 5 \mathrm{ft} ., 3 / 4 \mathrm{i} \\
4 \text { to } 5 \mathrm{ft} ., 5 / 8 \mathrm{t} \\
3 \text { to } 4 \mathrm{ft} ., 1 / 2 \mathrm{t}\end{array}$ & $\begin{array}{l}\text { up, No. } 1 \ldots \ldots \\
\text {, No. } 1 \ldots \ldots \ldots \\
\text {, No. } 1 \ldots \ldots \ldots\end{array}$ & $\begin{array}{l}\text { llows } \\
\text { allop } \\
\text { amble }\end{array}$ & $\begin{array}{rl}\$ 9 & 00 \\
8 & 00 \\
6 & 00\end{array}$ \\
\hline $\begin{array}{l}\text { lett } \\
\text { hess } \\
\text { rre de Anjou } \\
\text { Vernon } \\
\text { nce }\end{array}$ & $\begin{array}{l}\text { Clapp's Favorite } \\
\text { Buffum } \\
\text { Louise Bon } \\
\text { Seckel } \\
\text { Flemish Beauty }\end{array}$ & $\begin{array}{l}\text { Howe } \\
\text { Doyer } \\
\text { Tyson } \\
\text { Rutte } \\
\text { Wilde }\end{array}$ & Boussock \\
\hline
\end{tabular}

\section{NATIVE PLUM ON MARIANA.}

Code Word Per 100 Per 1000

$\$ 8000$

7000

5000

Two Years.

No. 1,5 to $6 \mathrm{ft} ., 3 / 4$ in. and up.............

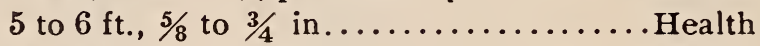

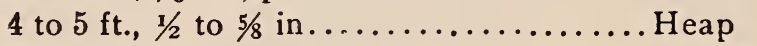

3 to $5 \mathrm{ft}$., mostly branched ............ Hoarse

One Year.

5 to $6 \mathrm{ft} ., 3 / 4$ in. and up...................

4 to $6 \mathrm{ft}$., $5 / 8$ to $3 / 4$ in...................

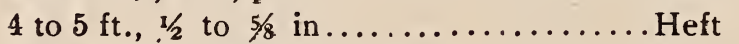

3 to 4 feet, mostly branched............ Help

Bartlett

Duchess

Mt. Vernon

3 to 4 feet, mostly branched................ Help
Sold

Per 100
$\$ 1300 \quad \$ 12500$
$1200 \quad 10000$

Sold

$1600 \quad 15000$

$1300 \quad 12500$

$1000 \quad 10000$

\section{PLUM ON PLUM-European Sorts.}

\section{Two Years.}

No. 1,5 to $6 \mathrm{ft} ., 3 / 4$ in and up.

No. 1,5 to $6 \mathrm{ft}$., $5 / 8$ to $3 / 4$ in............ Gather

No. 1,4 to $5 \mathrm{ft}$., $1 / 2$ to $5 / 8$ in............ Galvanic

No. 1,3 to $4 \mathrm{ft}$, mostly branched.
Bradshaw

Blue Damson

Empire

F. Damson

Fellenberg

Coe's Golden Drop.

German Prune
Gen. Hand

Genii

Grand Duke

Imperial

Lombard

Moore's Arctic

Niagara
Per 100

$\$ 1800 \$$

$\$ 1500 \quad 14000$

$1200 \quad 10000$

Per 1000

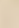

$800 \quad 7000$

Pond Seedling

Saratoga

Reine Claude

Smith Orleans.

Shipper's Pride.

Shropshire Damson

Yellow Egg. 


\section{JAPAN PLUM ON PEACH.}

\section{One and Two Years.}

No. 1,5 tc $6 \mathrm{ft}$., $3 / 4$ in. and up.

No. 1,4 to $5 \mathrm{ft}$, $5 / 8$ to $3 / 4$ in.

No. 1,4 to $5 \mathrm{ft}$., $1 / 2$ to $5 / 8$ in.....

$3 \mathrm{r} / 2$ to $5 \mathrm{ft}$., mostly branched.
Code Word

Garnet

Garnish

. Garrett

.Gathering
Per 100

$\$ 1600$

1500

1200

800
Per 1000

$\$ 15000$

13000

10000

7000

\section{VARIETIES OF NATIVE AND JAPAN PLUMS.}

Burbank

Botan

Desoto

Forest Garden

Golden Beauty

Hale

Hawkeye
Mariana

Milton

Ogon

Prunus Pissardii

Pottawatomie

Red June

Robinson
Satsuma

Simoni

Wolf

Weaver

Wild Goose

Willard

Wickson

\section{APRICOT ON PEACH.}

One Year and Two Years.

No. 1, 5 to $6 \mathrm{ft}$., $3 / 4$ in and up.

No. 1,4 to $6 \mathrm{ft}, 5 / 8$ to $3 / 4$ in

3 to $4 \mathrm{ft}$., mostly branched
Code Word

.Gavel

.Gazelle

.Gear

Gelatine
Per 100

$\$ 1100$

900

800

700
Per 1000

$\$ 10000$

8000

7000

6000
'Budd

Catherine

Superb
Early Golden

Gibb
Moore Park

Royal

\section{NECTARINE.}

One Year.

Code Word

.Gemmed

.Gender
Per 100

$\$ 1400$

1200
Per 1000

$\$ 13000$

10000

Boston

\section{QUINCE.}

Two Years.

Code Word

4 to $5 \mathrm{ft}$

3 to $4 \mathrm{ft}$

2 to 3

Champion

Meech's

Orange
.Harp

.Genesis

$\$ 1700$

1200

800
Per 1000

$\$ 15000$

12000

7000 


\section{GRAPES.}

Agawam, Code Word Per 100 Per 1000

$\$ 200 \$ 2000$

2 yrs., No $1 . . \ldots \ldots \ldots \ldots \ldots \ldots \ldots$................. $\quad 300 \quad 2500$

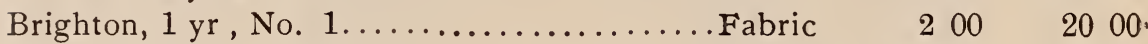

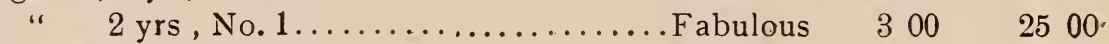

Concord, 1 yr , No. 1............................................. $150 \quad 1200$

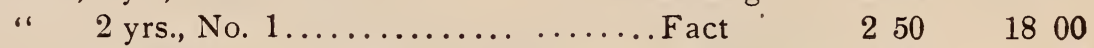

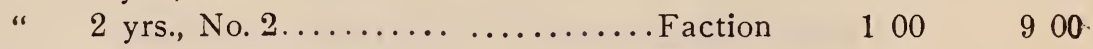

Campbell's Early, 1 yr., No.1.............Factor 710

Champion, 1 yr., No. 1.....................

" 2 yrs., No. 1.................Faculty. $300 \quad 25 \mathrm{c0}$.

Catawba, 1 yr,, No. 1 ................................... 20000

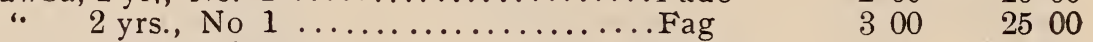

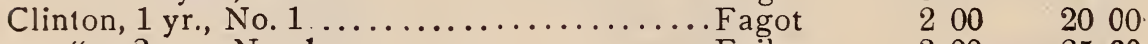

" 2 yrs., No. $1 \ldots \ldots \ldots \ldots \ldots \ldots \ldots \ldots \ldots \ldots \ldots$. . . . . . .

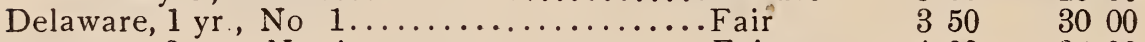

Elvira 2 yrs., No. L..................Faint $\quad 400 \quad 3500$

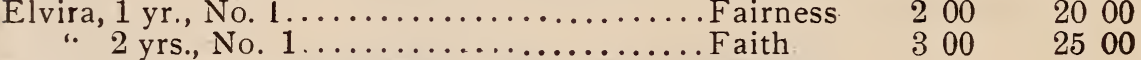

Empire State, 1 yr, No. $1 \ldots \ldots \ldots \ldots \ldots \ldots \ldots$. . . . . . 3 . 3 . $\quad 2500$

$350 \quad 3000$

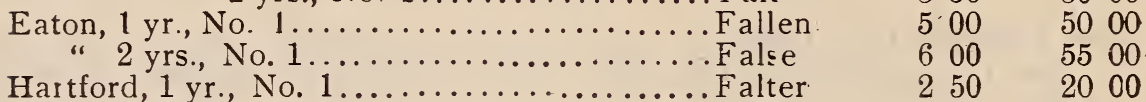

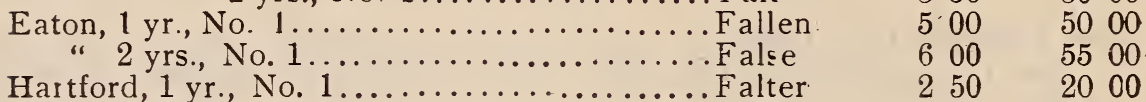

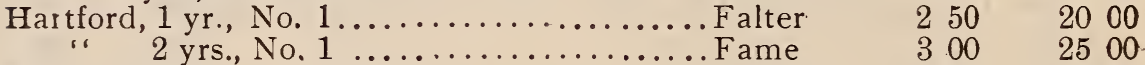

Ives' Seedling. 1 yr., No. 1 ............................ $250 \quad 2000$

" "2 yrs., No. 1...............Fashion $300 \quad 2500$

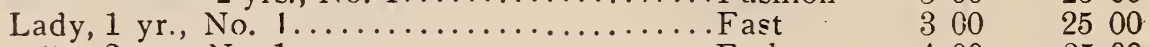

2 yrs., No. 1..................................... $400 \quad 3500$.

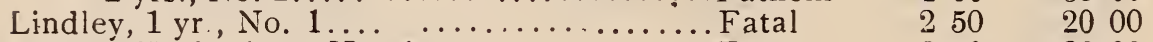

Moore's Early, 1 yr., No. 1.......................... $350 \quad 3000$

$400 \quad 40 \mathrm{CO}$

$350 \quad 3000$

$450 \quad 4000$

$250 \quad 2000$

$300 \quad 2500$

$3 \mathrm{CO} \quad 2500$

$350 \quad 3000$

$250 \quad 2000$.

$300 \quad 2500$

$250 \quad 2000$

$3.00 \quad 2500$

$250 \quad 2000$

$300 \quad 2500$

$200 \quad 20 \mathrm{CO}$

3. $00 \quad 25 \mathrm{CO}$

$350 \quad 3000$

4003500 .

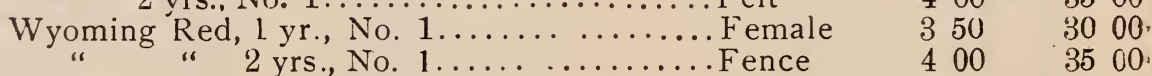

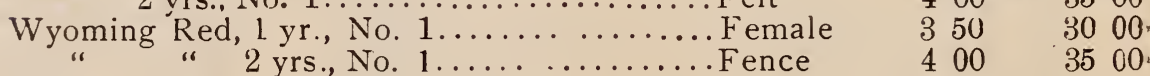

No. 2 Vines of the above assortment will be supplied at 70 per cent of the above prices. They are of good quality. Samples will be sent: by: mail on application 


\section{CURRANTS.}

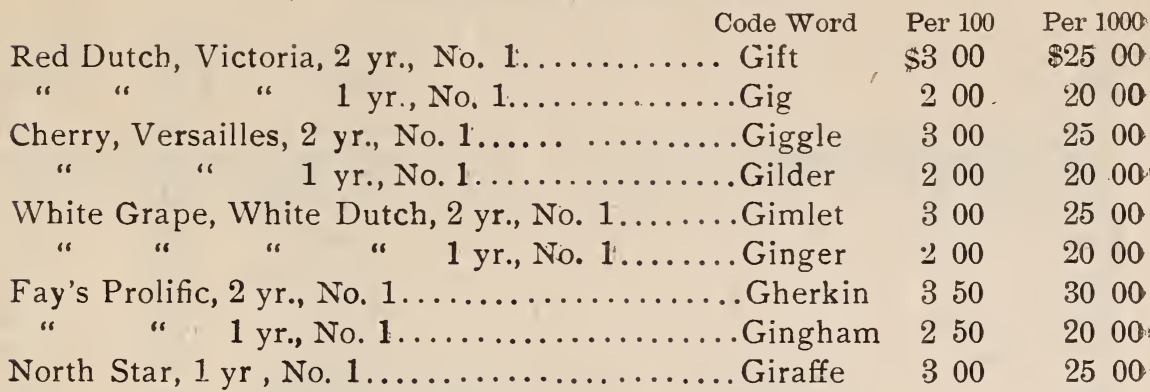

\section{GOOSEBERRIES.}

Code Word

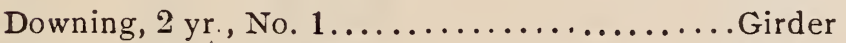

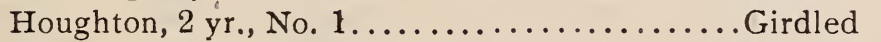

$\$ 300 \quad \$ 2500$

Smith's Improved, 2 yr., "No. 1..............Garbage

$250 \quad 2000$

Champion, 2 yr.. No. 1.

. Gang

3000

Industry, 2 yr., No. $1 . .$.

350

400

Chantauqua, 2 yr., No. 1

Girth

1000

Josselyn, 2 yr., No. 1

. Ginseng 1500

.Gizzard 500

\section{BLACKBERRIES.}

\begin{tabular}{|c|c|c|c|c|c|c|}
\hline \multirow{2}{*}{\multicolumn{3}{|c|}{ Early Harvest, root-cutting }} & \multirow{2}{*}{\multicolumn{2}{|c|}{$\begin{array}{l}\text { Code Word } \\
\text {. Glacial }\end{array}$}} & Per 100 & \multirow{2}{*}{$\begin{array}{l}\text { Per } 1000 \\
\$ 700\end{array}$} \\
\hline & & & & & & \\
\hline Snyder. & “ & “ & “ & ...Glacier & 100 & 1000 \\
\hline Erie & “ & “ & “ & ....Glad & 150 & 12 \\
\hline Kit & “ & “ & “ & ....Glair & 100 & 10 \\
\hline taruy, & "، & “ & “ & ...Gau & 100 & 10 \\
\hline at & “ & “ & “ & ...........Ganse & 300 & 25 \\
\hline ncient Britail & “ & “ & “ & ...........Gasconda & 150 & 12 \\
\hline
\end{tabular}

\section{RASPBERRIES.}

\begin{tabular}{|c|c|c|}
\hline . Code Word & Per 100 & Per 1000 \\
\hline Gregg, tips..... & $\$ \quad \dot{75}$ & $\$ 600$ \\
\hline$\ldots \ldots \ldots \ldots \ldots$ Halter & 75 & 600 \\
\hline 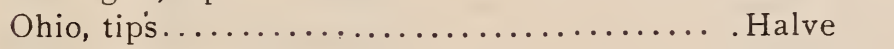 & 75 & 60 \\
\hline 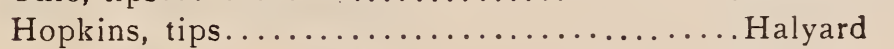 & 75 & \\
\hline Mammoth Cluster, tips........... & 75 & \\
\hline 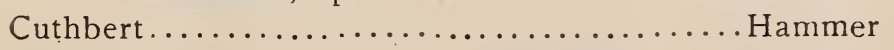 & 90 & 60 \\
\hline 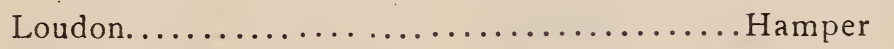 & 150 & 120 \\
\hline 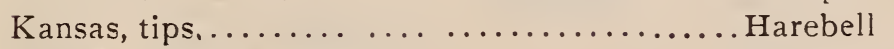 & 75 & \\
\hline Golden Queen.........................Harpoon & 100 & 100 \\
\hline $\operatorname{ardinal} \ldots \ldots \ldots \ldots \ldots \ldots \ldots \ldots \ldots$ Hammock & & \\
\hline
\end{tabular}


STRAWBERRIES.

\begin{tabular}{|c|c|c|}
\hline Code Word & Per 100 & Per 1000 \\
\hline ..Hand & $\$ 50$ & $\$ 300$ \\
\hline 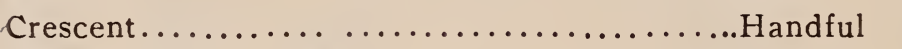 & 50 & 250 \\
\hline Cumberland Triumph.................Handily & 50 & 300 \\
\hline 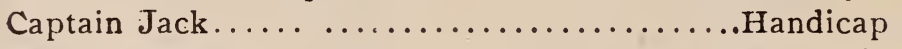 & 50 & 300 \\
\hline Chas. Downing..$\ldots \ldots \ldots \ldots \ldots \ldots$ & 50 & 250 \\
\hline 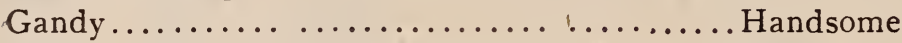 & 50 & 300 \\
\hline Parker Earle............................ & 50 & 300 \\
\hline 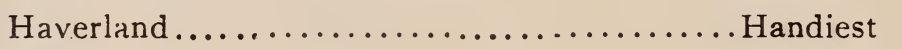 & 50 & 300 \\
\hline Jessie.......................Hangman & 50 & 300 \\
\hline Michael's Early.....................Hanker & 50 & 300 \\
\hline 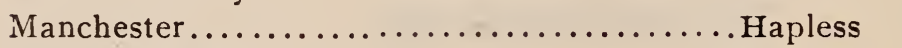 & 50 & 300 \\
\hline Miner's Prolific.....................Happen & 50 & 300 \\
\hline Greenville......................... Harvest & 50 & 300 \\
\hline 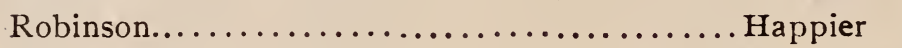 & 50 & 300 \\
\hline 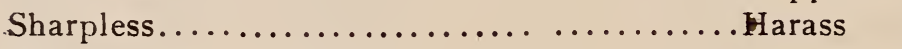 & 50 & 400 \\
\hline 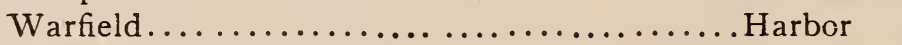 & 50 & 300 \\
\hline
\end{tabular}

\section{MISCELLANEOUS.}

Code Word

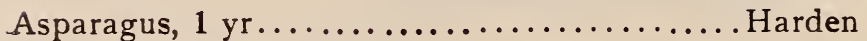

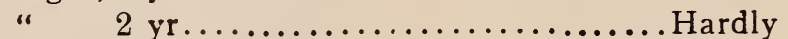

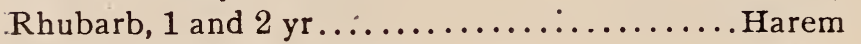

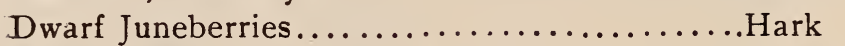

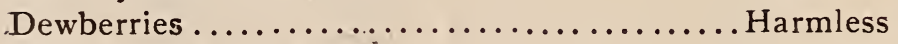

Strawberry Raspberry................... Gewgaw
Per 100

$\$ 40$

50

250

400

100

200
Per 1000

$\$ 350$

500

2000

3500

1000

1500

\section{PERSIMMONS.}

\begin{tabular}{|c|c|c|c|}
\hline American, & \begin{tabular}{cc} 
& \multicolumn{1}{c}{ Code Word } \\
$\ldots . \ldots . \ldots$. & $\ldots$ Hideous
\end{tabular} & $\begin{array}{l}\text { Per } 10 \\
\$ 200\end{array}$ & $\begin{array}{l}\text { Per } 100 \\
\$ 1500\end{array}$ \\
\hline “ & ............Higgle & 150 & 1000 \\
\hline “ & 2 to $3 \mathrm{ft} \ldots \ldots \ldots \ldots \ldots \ldots \ldots$ High & 100 & 800 \\
\hline ، & 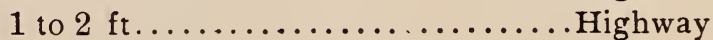 & 80 & 600 \\
\hline
\end{tabular}

\section{FIGS.}




\section{ORNAMENTAL DEPARTMENT.}

\section{ORNAMENTAL TREES.}

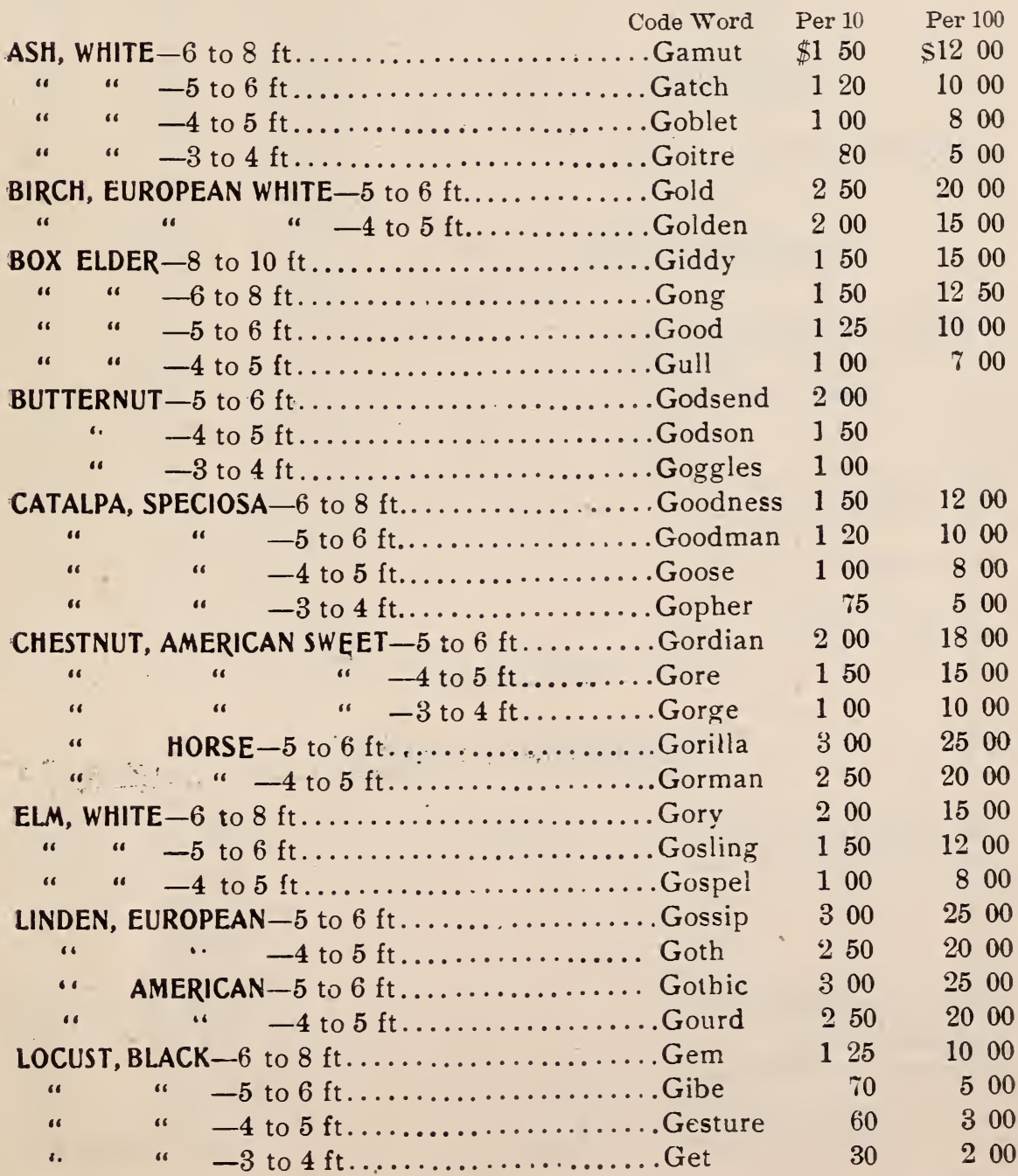




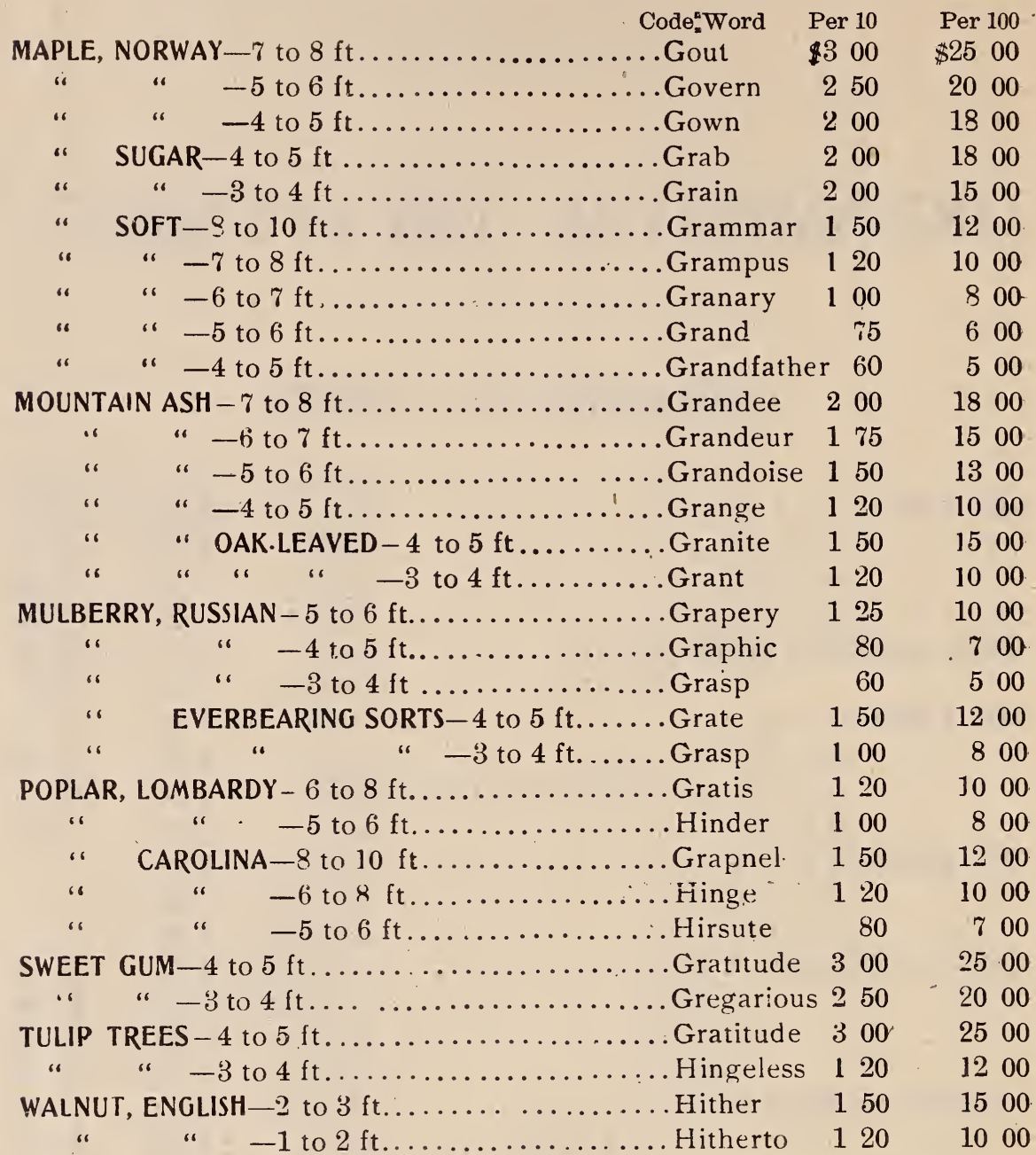

\section{WEEPING TREES.}

ASH, EUROPEAN MOUNTAIN $-2 y$ r. heads........... Gravel $\$ 500$ BIRCH, CUT.LEAVED -6 to $7 \mathrm{ft} \ldots \ldots \ldots \ldots \ldots \ldots \ldots \ldots$ Gravity 700

" " $\quad-5$ to $6 \mathrm{ft} \ldots \ldots \ldots \ldots \ldots \ldots \ldots \ldots$ Gravy 650

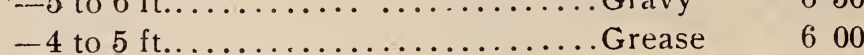

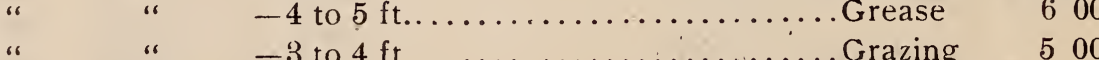

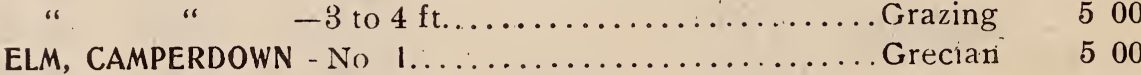

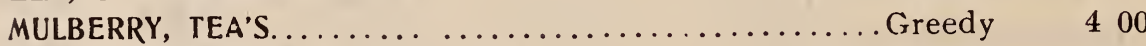

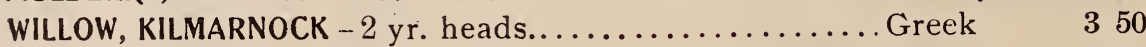

-1 yr. heads................... Green 250

NEW AMERICAN -1 yr. heads......................... 300 


\section{EVERGREENS.}

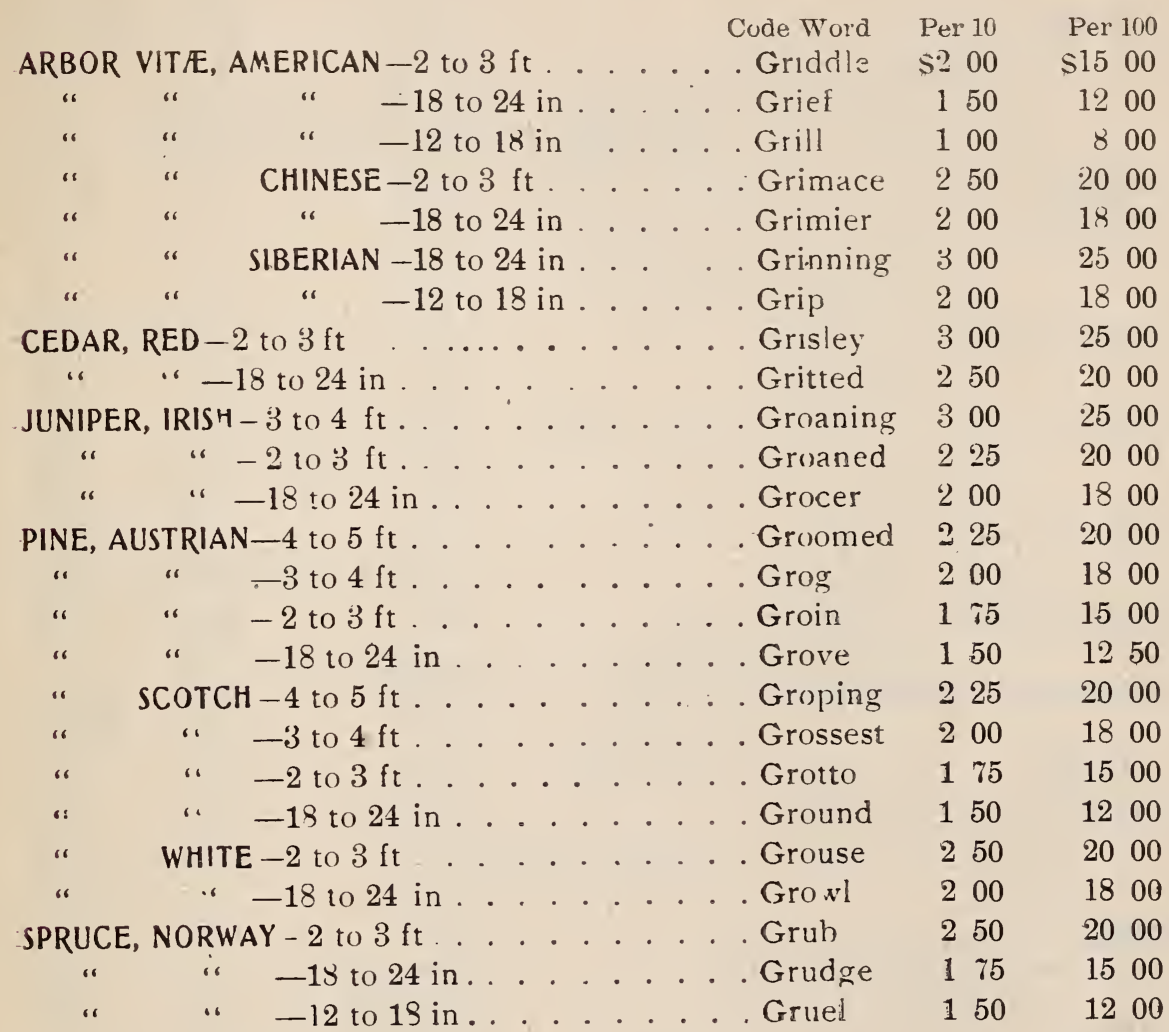

\section{CLIMBERS.}

\begin{tabular}{|c|c|c|c|c|c|c|}
\hline \multirow{3}{*}{\multicolumn{4}{|c|}{ 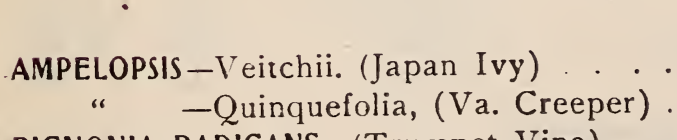 }} & \multirow{2}{*}{$\begin{array}{l}\text { Code Word } \\
\text {. . Halbred }\end{array}$} & \multirow{2}{*}{$\begin{array}{l}\text { Per } 10 \\
\$ 150\end{array}$} & \multirow[t]{2}{*}{$\begin{array}{l}\text { Per } 100 \\
\$ 1200\end{array}$} \\
\hline & & & & & & \\
\hline & & & & . . Hallelujah & 150 & \\
\hline BIGNONI & IA RADICANS - (Trumpet & Vine) .. & & Ham & 100 & \\
\hline & ii, strong. . & & & & & \\
\hline “ & strong... & & & . . Hack & 150 & \\
\hline “ & $-\mathrm{Fla}$ & & & .. $\mathrm{Ha}$ & 150 & \\
\hline “ & -Paniculata... & . . & & ude & 12 & \\
\hline NEYS & UCKLE-Hall's Japan.. & $\cdots$ & & & 100 & \\
\hline & green ... & & & . . $\mathrm{Ha}$ & 100 & \\
\hline “ & -Canadian. & & & . . $\mathrm{H}$ & 100 & \\
\hline “ & -Chinese. & & & . Halcyon & 100 & \\
\hline & . . & . . . & & . Halloo & 100 & \\
\hline
\end{tabular}




\section{DECIDUOUS SHRUBS.}

Code Word

ALTHEAS-Double, 3 to $4 \mathrm{ft}$. . . . . . Guano

- -Double, 2 to $3 \mathrm{ft}$. . . . . Guard

“- - Variegated Leaved 18 to 24 in ; . Guave

" " " 15 to 18 in. . Geranium

ALMOND -Double Flowering, 18 to 24 in . . Guess

BERBERRY--Purple-Leaved, 18 to 24 in . . ..Guide

" -Purple-Leaved, 12 to 18 in . . Guild

“ - Thunbergii 18 to 24 in..... Guilty

CALYCANTHUS-18 to 24 in . . . . . Guinea

" -12 to 18 in ....... Gulse

DUETZIA-Pride of Rochester, 18 to 24 in . . Gulch

"-Fortunea ........... . Guitar

" - Crenata, Fl. P., 12 to 18 in .... . Gulf

FRINGE-Purple 2 to $3 \mathrm{ft}$. . . . . . . Gullett

"-Purple, 18 to 24 in ....... Gullied

HONEYSUCKLE-Upright, 2 to $3 \mathrm{ft}$. . ... . Gulp

$$
\text { " -Upright, } 18 \text { to } 24 \text { in . . . . Gummy }
$$

HYDRANGEA-GRANDIFLORA-2 to $3 \mathrm{ft}$. . . . Gentle

$$
\text { “ }-18 \text { to } 24 \text { in. . . . Gunnery }
$$

Per 10 Per 100

$\$ 120 \quad \$ 1000$

$100 \quad 850$

$125 \quad 1200$

100.1000

$125 \quad 1200$

$75 \quad 700$

$70 \quad 500$

$100 \quad 800$.

$100 \quad 1000$

$80 \quad 800$

$100 \quad 800$.

$100 \quad 800$

$100 \quad 800$.

$100 \quad 1000$.

$80 \quad 800$

$100 \quad 800$.

$80 \quad 800$

$150 \quad 1200$

$120 \quad 1000$.

JAPONICA - or Japan Quince, 18 to 24 in. . . Galena $\quad 100 \quad 800$.

LILACS-Purple, 18 to 24 in . . . . . . . Gunwale

$100 \quad 800$

" - White, 18 to 24 in . . . . . . . Gurgle 1251000

" -2 to $3 \mathrm{ft}$........... Gainsay 1501000

PRIVET-Common, 12 to 18 in . . . . . Gush $70 \quad 500$

"-California, 18 to 24 in ...... Gymnastics $70 \quad 500$

"-California, 12 to 18 in ..... Generous $50 \quad 300$.

PRUNUS TRILOBA-2 to $3 \mathrm{ft}$. . . . . . . Grangreen 1501000

SYRINGA-(Mock Orange) 18 to 24 in . . . Gussett I $00 \quad 800$

"-(Mock Orange) 12 to 18 in . . . . Gusto

SPIREA-Billardi, 18 to 24 in . . . . . . . Gutted

"-Van Houtei, 18 to 24 in ... Guttering

"-Opulifolia Aurea, 12 to 18 in . ... Guzzle

SNOWBALLS -2 to $3 \mathrm{ft}$. . ....... Gymnast

“ -18 to 24 in ........ . Gypsum

WEIGELIA-Five sorts, 18 to 24 in . . . . Gypsy

"-Five sorts, 12 to 18 in . .... Gyrate

$80 \quad 700$

$100 \quad 800$

$125 \quad 1000$

$150 \quad 1200$

$\begin{array}{llll}1 & 25 & 10 & 00\end{array}$

$100: 1000$

$125 \quad 1000$

100800 .

\section{ROSES.}

HYBRID PERPETUALS-2 yr., and extra 1 yr. strong. Hasten " " -1 y r., No. 1 ...... Hastily

MOSS-2 yr., and extra'1 yr. strong ...... . Hat

" -1 yr., No. 1........ Hatch

CLIMBING-2 yr., and extra 1 yr. strong ... . Hatchet

" -1 yr., No. 1....... Hateful

CRIMSON RAMBLER-2 yr., No. 1 . . . . Hatred

YELLOW RAMBLER-2 yr., No. 1 ...... . Grudy

Per 100

Per 1000

$\$ 1200 \$ 10000$.

$1000 \quad 9000$

$1200 \quad 10000$

10009000 ,

$800 \quad 7000$

$800 \quad 7000$

100010000 .

TREE ROSES ................ Gauge

1200

5000 


\section{SEEDLINGS AND SCIONS.}

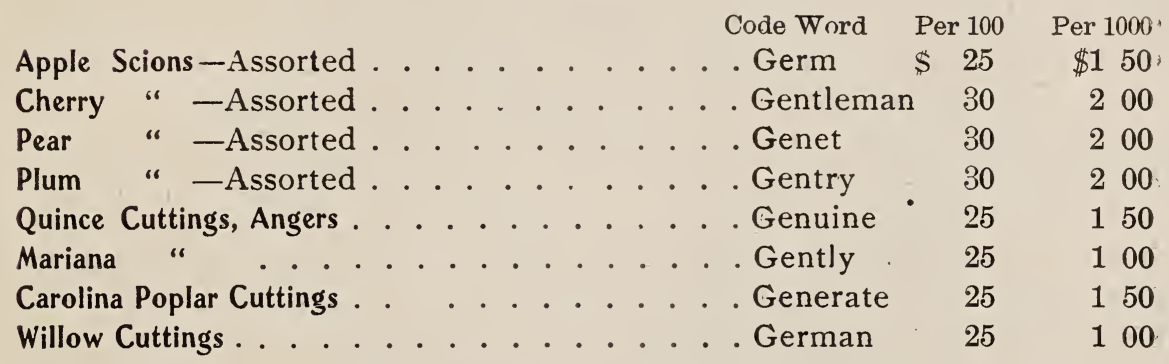
quality.

Our Apple Scions are from a young scion orchard, and are of good

\section{SEEDLINGS.}

Pear, French, 3-16 in. and all up . . . . . . . Gland \$6 50

" " $2-16$ to $3-16$ in.......... Glanders 450

"Keiffer, 3-16 in. and all up........ Glass 600

" " $2-16$ to $3-16$ in .......... Gladiator 450 ,

" " under 2-16 in .......... Gleam 250 ;

Cherry, Mahaleb, 3-16 in. and up ........ 60

" " $2-16$ to $3-16$ in........... 400

" " under $2-16$ in.......... 250 .

\section{APPLE SEEDLINGS.}

1 yr., No. 1, 3-16 and all up, straight .......... Prices quoted:

1 yr., No. 1, 2-16 to 3-16, straight . . . . . . . . . on

1 yr., No. 1, 3-16 and all up, branched . . . . . . . application.

1 yr., No. 1, 2-16 to $3-16$, branched ............

1 yr., under 2-16

Apple Grafts . 


\section{FOREST TREE SEEDLINGS.}

All Nursery=Grown.

Code Word Per 1000

Russian Mulberry, $3 \mathrm{ft}$. and up ......... . Glen

$\$ 500$

" " 1 yr., 18 to 24 in......... Glide 250

" " $1 \mathrm{yr}, 12$ to $18 \mathrm{in.......} \mathrm{Glimmer} 200$

" " 1 yr, 8 to 12 in......... Glisten 125

" " 1 yr., less than 8 in ....... Glitter 100

Ash, 1 yr., No. 1,12 to 18 in ........... Glorious 200

"6 to 10 in ................. Gloss 150

Catalpa, 1 yr., No. 1,18 to 24 in.......... Glottis 250

" 1 yr., No. 1,12 to 18 in........... Glove 200

" 8 to 12 in.............. Glorify 150

Black Locust, 1 yr, 3 to $4 \mathrm{ft}$........... Gild 500

" " $1 \dot{\mathrm{y} r}, \mathrm{2}$ to $3 \mathrm{ft}$........... Glue 250

" " 1 yr., No. 1,18 to 24 in....... Glutton 225

" " 1 y.r., No. 1,12 to 18 in........ Gnarl 175

" " 1 yr., No. 1,8 to 12 in......... Gnarled 200

Honey " 12 to 18 in ............ Glossary 300

" " 8 to 12 in........... Gneiss 250

Box Elder, 1 yr., No. 1, 18 to 24 in.......... Gatch 250

" " 1 yr., No. 1,12 to $18 \mathrm{in........} \mathrm{Gobbler} 200$

" " 1 yr., No. 1,8 to 12 in........ Goatee 150

Maple, 1 yr., 2 to $3 \mathrm{ft}$.............. Gnaw 200

" 1 yr., 18 to 24 in........... . Gnat 175

" 1 yr., 12 to 18 in . . . . . . . . . . Gnome 150

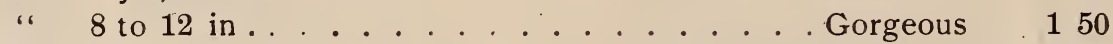

Osage Hedge, 1 yr., No. 1. . . . . . . . . Goad 125

No. 2........... Glycerine 80

\section{HERBACEOUS PLANTS AND BULBS.}

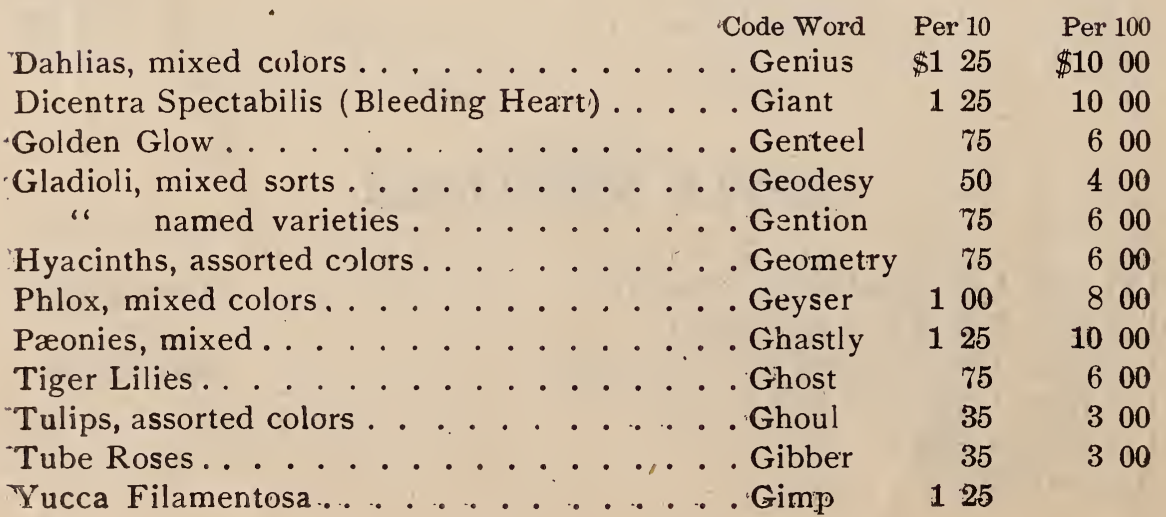

